\title{
Mediastinal Atlas Creation from 3-D Chest Computed Tomography Images: Application to Automated Detection and Station Mapping of Lymph Nodes
}

\author{
Marco Feuerstein $^{\mathrm{a}, \mathrm{b}}$, Ben Glocker ${ }^{\mathrm{b}, \mathrm{c}}$, Takayuki Kitasaka ${ }^{\mathrm{d}}$, Yoshihiko Nakamura ${ }^{\mathrm{a}}$, Shingo Iwano ${ }^{\mathrm{e}}$, Kensaku Moria, \\ ${ }^{a}$ Graduate School of Information Science, Nagoya University, Nagoya, Japan \\ ${ }^{b}$ Computer Aided Medical Procedures (CAMP), Technische Universität München, Munich, Germany \\ ${ }^{c}$ Microsoft Research, Cambridge, UK \\ ${ }^{d}$ Faculty of Information Science, Aichi Institute of Technology, Toyota, Japan \\ ${ }^{e}$ Graduate School of Medicine, Nagoya University, Nagoya, Japan \\ ${ }^{f}$ Information and Communications Headquarters, Nagoya University, Nagoya, Japan
}

\begin{abstract}
One important aspect of lung cancer staging is the assessment of mediastinal lymph nodes in 3-D chest computed tomography (CT) images. In the current clinical routine this is done manually by analyzing the 3-D CT image slice by slice to find nodes, evaluate them quantitatively, and assign labels to them for describing the clinical and pathologic extent of metastases. In this paper we present a method to automate the process of lymph node detection and labeling by creation of a mediastinal average image and a novel lymph node atlas containing probability maps for mediastinal, aortic, and N1 nodes. Utilizing a fast deformable registration approach to match the atlas with CT images of new patients, our method can maintain an acceptable runtime. In comparison to previously published methods for mediastinal lymph node detection and labeling it also shows a good sensitivity and positive predictive value.
\end{abstract}

Keywords: Probabilistic Atlas, Mediastinum, Lymph Node Map, Lung Cancer, Deformable Registration

\section{Introduction}

With estimated 1.3 million cases per year, lung and bronchus cancer is the leading cause of cancer death worldwide [1]. To prognosticate and determine the suitability of cancer patients for surgical resection or alternative ways of treatment, accurate mediastinal staging needs to be performed. Before invasive histological staging investigations such as transbronchial needle aspiration can be performed, it is generally agreed that a noninvasive (usually contrast enhanced) computed tomography (CT) scan of the chest is acquired [2]. In the current clinical routine, the localization, quantification, and characterization of lung tumors needs to be performed manually by examining all CT slices. To automate this time-consuming process and support the physician as much as possible, various computer assisted techniques have been proposed, in particular for pulmonary nodule detection and analysis [3].

Since mediastinal lymph nodes are involved for about $30 \%$ to $44 \%$ of diagnosed lung cancer patients [4], one important aspect of lung cancer staging is the determination of the zones and stations (cf. Fig. 1), shapes, and sizes of these nodes. Carefully analyzing enlarged lymph nodes, a first estimation of lung cancer spread can be made and target regions and margins for surgical resection or other means of treatment can be defined [5]. However, it should be noted that even though the general guidelines specify lymph nodes to be pathological if their short axis is greater or equal than one centimeter [6], a differentiation of malignant from benign mediastinal lymph nodes by size alone may not be reliable [7, 8]. Furthermore, the benefit of contrast administration during $\mathrm{CT}$ has been challenged for lymph node detection [9], in particular for mediastinal lymph nodes [10].

In contrast to the large amount of work done on the automated analysis of the lung such as detection, quantification, and classification of pulmonary nodules, emphysema, and pulmonary embolisms [3], little work on the automated analysis of the mediastinum and mediastinal lymph nodes has been published to date. The publications can be mainly categorized into detection $[12,13,14,15,16]$ and segmentation $[17,18,19,20,21,22,23,24]$ of lymph nodes, labeling of lymph node stations [25, 26], and computer assisted treatment planning [27]. For the segmentation of lymph nodes, recently also a commercial system has been announced [28].

Although the results of the proposed lymph node segmentation methods are promising, they all require manual interaction, i.e. at least one mouse click in the center of each lymph node to be segmented or its initial manual delineation, before e.g. a mass-spring model [22] or deformable model [23] can be iteratively updated to fit the node. Automated detection techniques aim to tackle this problem and attempt to find lymph nodes without any user interaction. In [13], detection is achieved by template matching in the Fourier space. This is done after a manual definition of the lymph node intensity range that was adjusted for each experimental dataset by means of a previously obtained gold standard segmentation. This manual initialization is not required in [14], where three-dimensional minimum directional difference filtering is applied to highlight voxels, which are brighter than their surrounding voxels on a sphere 


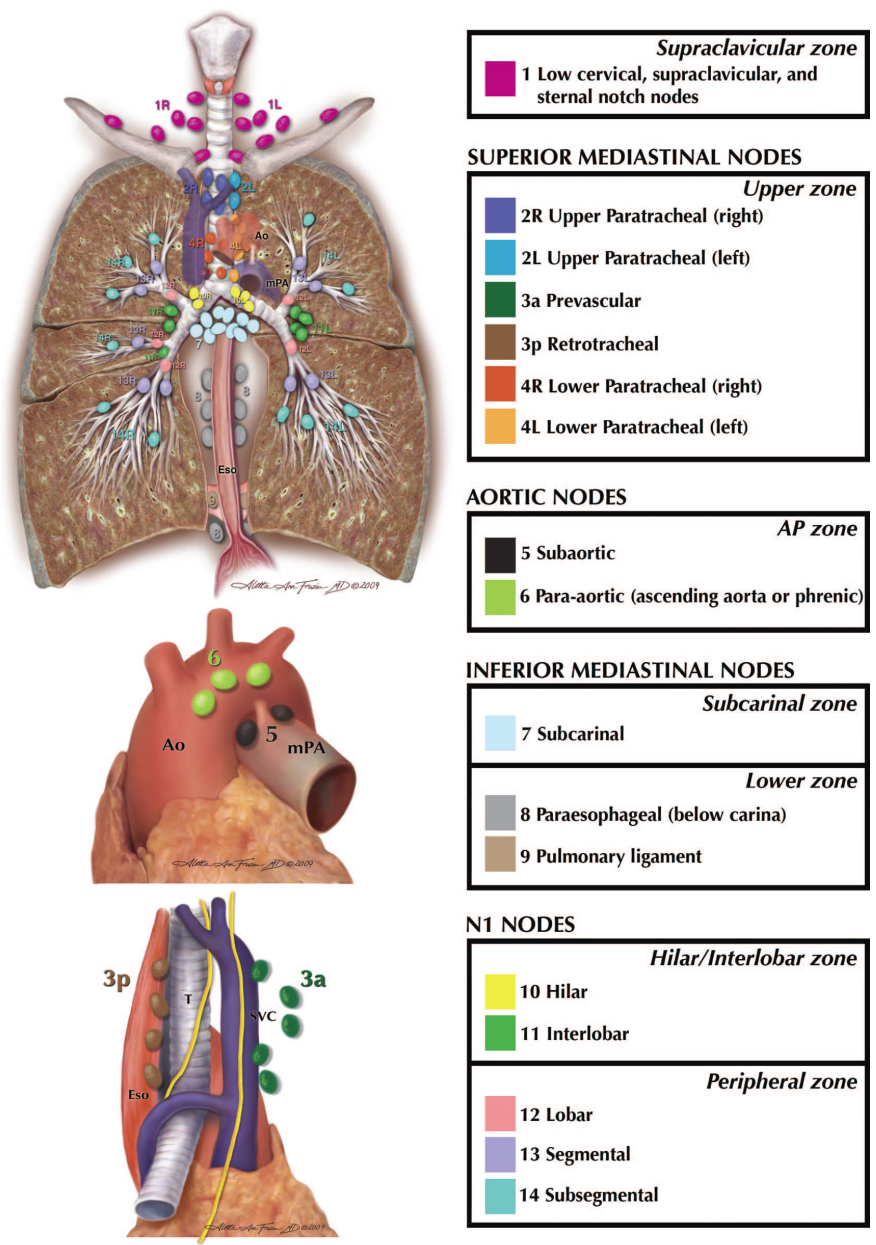

Figure 1: The International Association for the Study of Lung Cancer (IASLC) lymph node map. Image courtesy of Valerie W. Rusch [11].

surface. As a lot of voxels need to be compared for this method, it had a runtime of about two to three hours per CT dataset.

Most of the detection techniques still have a low positive predictive value, i.e. detect many other structures such as musculature and vasculature as false positive nodes $[13,14,16]$. To reduce this number of false positives, a restriction of the search space can be effective, i.e. a limitation to certain parts of the CT image where the detection technique is searching for lymph nodes. This was exemplarily shown for enlarged neck lymph nodes in one head data set with a manually restricted search map [15], where mass spring models are seeded into the search map at equal distances and tried to be fit to lymph nodes. The fitting quality can then be assessed according to a quality measure embedded into the model.

When we consider automated labeling of mediastinal, aortic, and N1 lymph nodes inside the thorax according to Fig. 1, before the actual labeling process begins, anatomical key structures such as airways, aorta, pulmonary artery, and sternum need to be detected to define the nodal stations [25, 26], which itself can be time-consuming and error-prone. Using the airways and the aorta as anatomical landmarks and machine learn- ing to train the labeling process, the best achievable station labeling accuracy to date was $76 \%$ [25].

To improve the precision and accuracy of lymph node detection and labeling, we here propose to utilize deformable registration to generate an unbiased average mediastinal image from a patient database and its corresponding mediastinal probabilistic lymph node atlas from ground-truth lymph node segmentations of this database. This atlas can be used efficiently in mediastinal lymph node detection to restrict the search space for possible lymph node candidates and in station labeling to accurately assign stations to newly detected lymph nodes.

Atlases have been proposed to be utilized for several applications in various parts of the human body. Most frequently they are used in brain imaging to support tasks like segmentation [29] and functional mapping [30]. Also efforts of lung atlas creation have been made to establish regional ranges of normative values for lung structure and function [31].

Another emerging application field of atlases is the estimation of target areas and volumes for the planning of radiation therapy to head and neck, thoracic, pelvic and other lymph nodes [32, 33, 34, 35, 36, 37, 38, 39, 40]. To create such an atlas, first lymph node regions are labeled manually in one image $[35,37]$ or a set of database images [32]. These labels are then transferred into a target image after registration of the target and database images. For registration, various methods have been used such as landmark-based [33], hybrid landmark-based and nonlinear [34, 36], hybrid linear and nonlinear [38, 39], and active contour based [40] techniques.

However, to date no fast and automatic method for the creation of a mediastinal lymph node atlas was developed, which is able to handle the high inter-patient variability of lymph node locations. At the same time it needs to be robust against cardiac motion artifacts and contrast intensity differences apparent in chest CT. Our work aims at filling this gap.

In summary, to deal with the above issues, we here present a fast and automatic method utilizing deformable registration for the creation of a probabilistic mediastinal lymph node atlas. We successfully apply this atlas to improve the precision and accuracy of currently available techniques for the automated detection and labeling of mediastinal, aortic, and N1 lymph nodes of almost any size in both contrast enhanced and non-contrast chest CT.

\section{Method}

In the following, we detail all steps necessary to create the probabilistic mediastinal lymph node atlas and its application to lymph node detection and labeling. As both detection and labeling require a dense distribution of lymph nodes labeled in the atlas, but the location and number of lymph nodes within nodal zones is highly variable from patient to patient, we decided to gather all available information in one single atlas. That is, if we have a set of CT images from our database with manually delineated lymph nodes labeled according to their station number [11], we transform all lymph node labels into the same atlas. This is opposed to atlas based segmentation techniques 
that usually have a one-to-one mapping of the structures to be delineated and in general work best when utilizing multiple individual atlases [29].

If we now want to detect and label lymph nodes in a new CT image, we simply transform the atlas labels into this new CT image. To obtain the necessary transformation between the atlas and the new CT image, from the set of CT images of our database we once create an average mediastinal image, which is defined in the same coordinates as the atlas, and register this average image to the new CT image.

Since the average image should represent an average mediastinal patient image, it needs to be unbiased and contain both average intensity and average shape information from all CT images of our database. To achieve that, we generate it iteratively by performing a series of deformable registrations minimizing the intensity and shape difference between itself and all database images, as outlined in Sec. 2.2. Sec. 2.1 describes the deformable registration method that we utilize during the average image creation and for any new CT image that we want the average image to be registered to. Once the average image and hence its corresponding atlas is registered to a new CT image, we can transform the atlas labels into the new CT image and perform our lymph node detection and station labeling techniques, as detailed in Sec. 2.3.

Figure 2 summarizes all steps necessary for the lymph node detection and station mapping process, including its preceding average image and atlas creation, which is performed once offline.

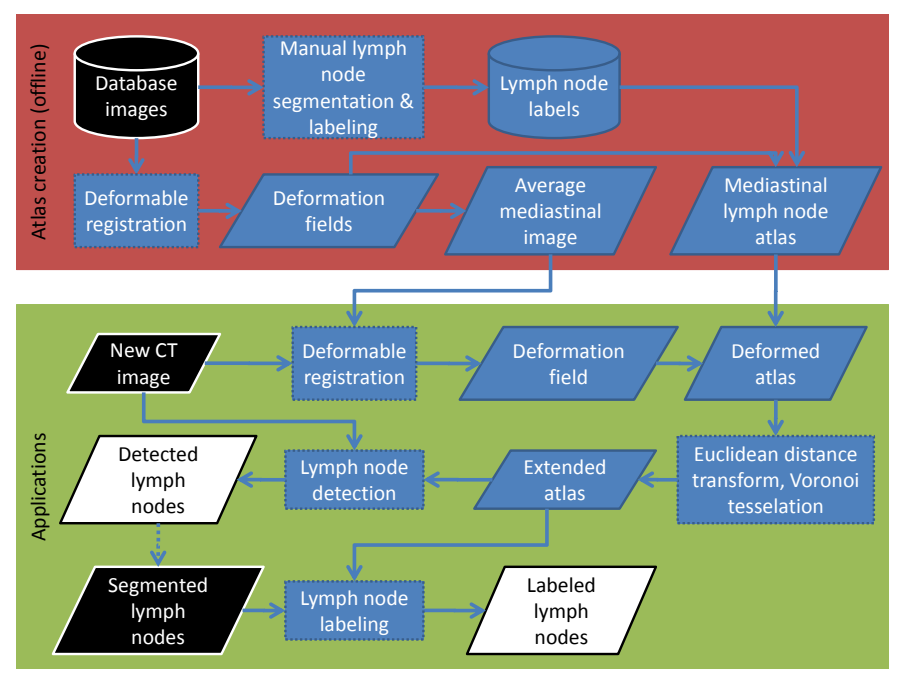

Figure 2: Flowchart of all steps performed for atlas creation, detection, and station mapping of mediastinal lymph nodes. Rectangles represent processes, parallelograms represent data. Input images are marked black, output is marked white. Note that the input of lymph node labeling can be either the lymph nodes automatically detected by our method or manually segmented ones. This is shown by the dotted arrow.

\subsection{Deformable Registration}

We use a recently proposed intensity-based deformable registration method [41] based on free-form deformations (FFDs) [42] and discrete Markov random field (MRF) optimization. We consider two different similarity measures for the registration of CT images. Since we are dealing with contrast enhanced and non-contrast $\mathrm{CT}$ registration, we decided to use mutual information [43, 44, 45] and normalized cross correlation [46]. We find that both measures give sufficient registration results as presented later in Sec. 3.

\subsection{Atlas Creation}

We generate the probabilistic mediastinal lymph node atlas in three steps. First, we globally align all database images at the carina tracheae. Next, all database images are utilized to iteratively generate an unbiased average mediastinal image. Finally, all manually delineated lymph node labels corresponding to the database images are transformed into the probabilistic lymph node atlas defined in the same coordinates as the average image. Fig. 4 illustrates parts of the atlas generation process.

\subsubsection{Global Image Alignment}

To initialize the registrations required for average image creation, we first align all database images at the carina tracheae by a simple translation, as the carina holds a central position within the mediastinum. It can easily be extracted by analyzing a segmentation of the airways that can be obtained fullyautomatically by any technique proposed in the literature [47]. We here use a method developed by our group [48, 49].

To simplify the following image registration process, we also resample and resize all database images to the same dimensions and to isotropic resolution of $1 \mathrm{~mm}$. We chose $1 \mathrm{~mm}$ to keep a good balance between reasonable size and sufficient details, both affecting the runtime and results of the deformable registration process. Furthermore, we threshold all images to be within the same intensity range $[-1000,1000]$ Hounsfield units (HUs) to deal with potential imaging artifacts. This is beneficial for the creation of histograms required for mutual information calculation.

\subsubsection{Average Mediastinal Image}

Several methods for the creation of an image containing average intensity and average shape information have been proposed within the scope of brain atlas development [50, 51, 52]. Our method for average mediastinal image creation is inspired by one of these works [50]. The basic idea is to register all database images to a fixed image (arbitrarily chosen from the database images), which is iteratively updated until it converges towards the final average image.

Figure 3 depicts the steps necessary to generate the average image $I_{i}$ in the $i$ th iteration. Unlike [50] we utilize the deformable registration technique introduced in Sec. 2.1 to generate all deformation fields between the database images and the fixed image, with mutual information and normalized cross correlation, respectively, as cost function. So in each iteration $i$ we will perform as many registrations as we have images in our 


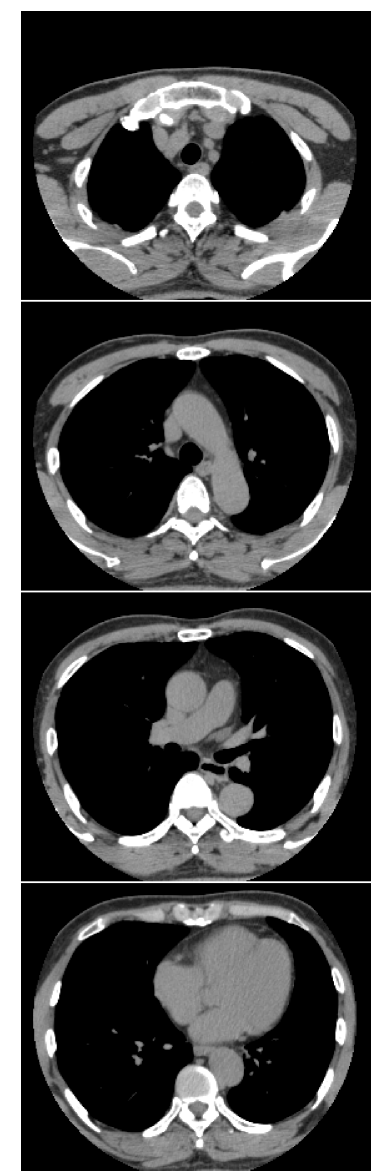

(a) $i=0$ (initial fixed image)

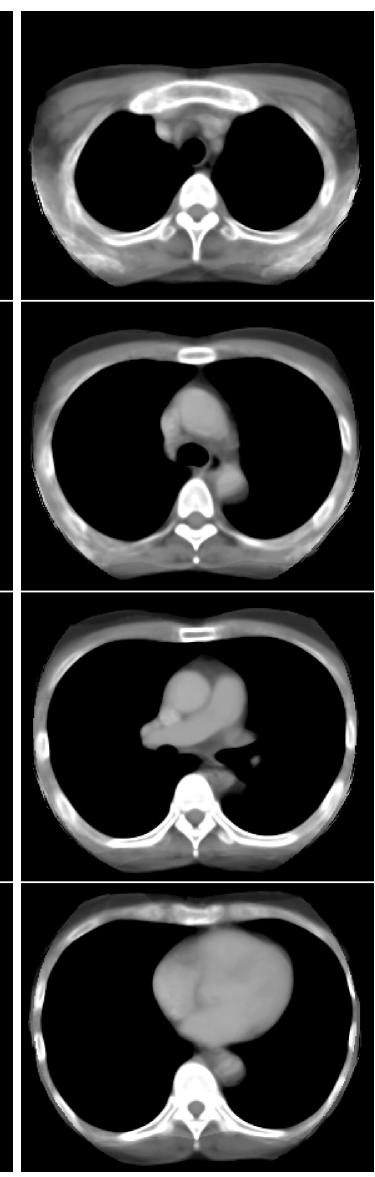

(b) $i=1$

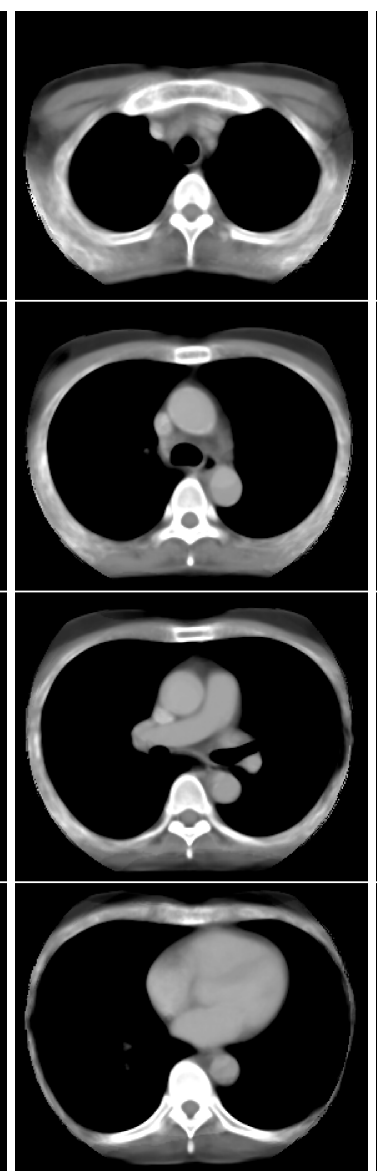

(c) $i=4$

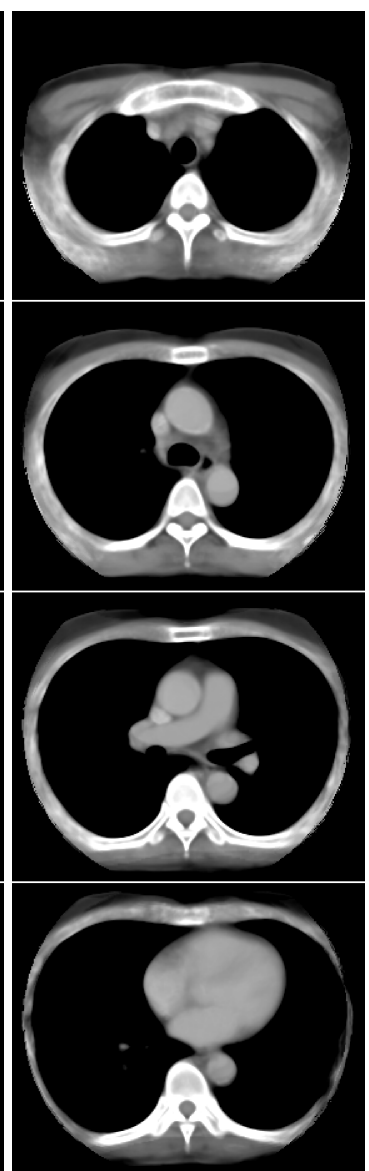

(d) $i=9$ (final average image)

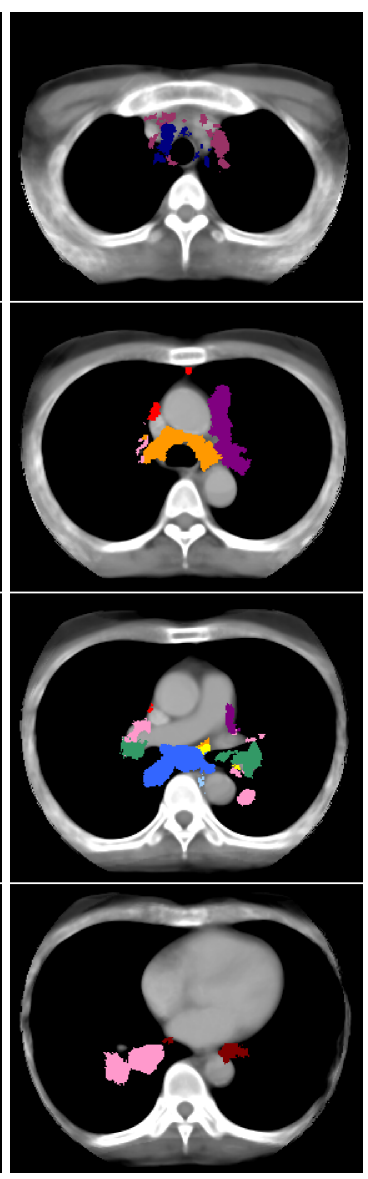

(e) $i=9$ (final atlas)

Figure 4: Four exemplary axial slices (each row showing the same slice number) during atlas generation for selected iterations $i$, from the initial fixed image to the final atlas. Different colors represent different lymph node stations.

database. To compute the inverse average deformation field, we apply a forward resampling approach [53]. However, we here deal with rather large deformations leading to sporadic holes in the inverse deformation field. We fill these holes by trilinear interpolation of the neighboring voxels ${ }^{1}$ and not by mathematical morphology, as proposed in [53]. The average image generation is considered completed if

$$
\begin{aligned}
\operatorname{NID}\left(I_{i}, I_{i+1}\right) & \geq \operatorname{NID}\left(I_{i-1}, I_{i}\right) \\
\vee \quad 0.001 & \geq\left\|\mathrm{NID}\left(I_{i}, I_{i+1}\right)-\operatorname{NID}\left(I_{i-1}, I_{i}\right)\right\|,
\end{aligned}
$$

where

$$
\operatorname{NID}\left(I_{m}, I_{n}\right)=\sqrt{\frac{\sum_{\boldsymbol{x}}\left(I_{m}(\boldsymbol{x})-I_{n}(\boldsymbol{x})\right)^{2}}{\sum_{\boldsymbol{x}}\left(I_{m}(\boldsymbol{x})\right)^{2}}}
$$

is the normalized intensity difference between image $I_{m}$ and $I_{n}$.

It has been shown in [50], that the resulting average image does not depend on the initial selection of the fixed image.

${ }^{1}$ Please note that in case of holes bigger than 1 voxel we just fill the holes from the outside to the inside, interpolating between all remaining (at least one) neighboring voxels.

\subsubsection{Probabilistic Lymph Node Atlas}

Once we have the average mediastinal image and the deformation fields from the database images into the average image, we apply these deformation fields to all lymph node labels delineated in the database images. In this way, we can generate a single lymph node atlas accumulating all the information about lymph node locations from the database images. During atlas generation, we assign the label (representing a lymph node station number) of a transformed voxel to the corresponding atlas voxel. If multiple different labels are assigned to the same atlas voxel, we simply select the most often occurring label.

\subsection{Applications}

If we want to utilize the probabilistic atlas in any new CT image to detect or label lymph nodes, we only need to register the average image with this new CT image by applying global image alignment (Sec. 2.2.1) and deformable registration (Sec. 2.1) and transform the lymph node atlas accordingly.

Atlas Extension. However, as the atlas only represents the lymph node distribution of a limited set of ground-truth segmentations, we also virtually extend the atlas. We do this by generating a Voronoi map [54] of the original atlas, so we can 


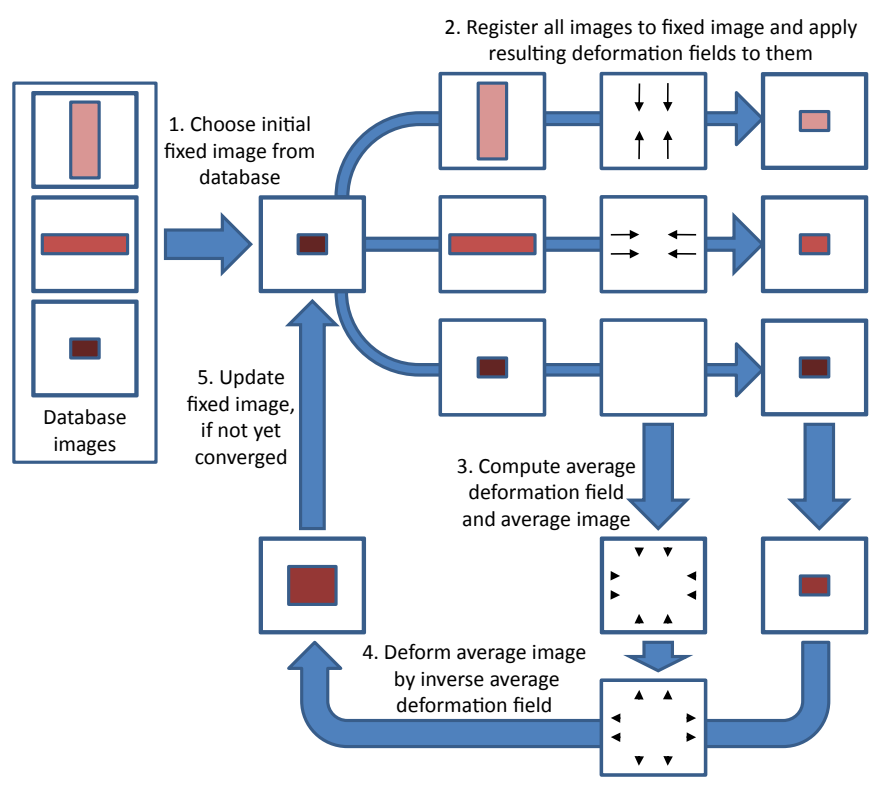

Figure 3: Average mediastinal image creation steps. The black arrows indicate the deformation fields.

also assign a lymph node station label to every non-labeled voxel of the atlas. If we additionally compute a map of Euclidean distances to the nearest originally labeled atlas voxels, we can optionally extend the atlas labels based on these distances and the Voronoi map. For instance, a $2 \mathrm{~mm}$ extension means that every voxel within $2 \mathrm{~mm}$ of the originally labeled atlas voxels gets the same label as specified in the Voronoi map, every voxel further away than $2 \mathrm{~mm}$ gets no label.

Figure 5 visualizes these possible atlas modifications. In detail, Fig. 5c shows an exemplary Voronoi map colored according to different lymph node stations. Even if the obtained lymph node atlas does not completely cover particular lymph node stations, the Voronoi map enables us to easily label every voxel of an image, which is important for lymph node station mapping (see Sec. 2.3.2). If we combine the Voronoi map with a Euclidean distance map (cf. Fig. 5d) of the atlas, we can also arbitrarily extend the atlas, as shown in Fig. 5e. This extension is useful during lymph node detection (see Sec. 2.3.1), as we know that the original atlas may not contain all individual lymph nodes of a patient, but is already very close to them.

\subsubsection{Lymph Node Detection}

As already mentioned in the introduction, lymph node detection techniques presented to date usually lack a satisfying positive predictive value, i.e. many false positive lymph nodes are still detected. Using an atlas we can naturally restrict the search space for possible lymph node candidates. We here outline the procedure developed by our group to detect lymph nodes [16] and incorporate our new probabilistic mediastinal lymph node atlas.

The method basically performs Hessian analysis at multiple scales to detect blob-like structures and applies a series of steps to reduce the number of false positive lymph nodes. As opposed to our previous work [16], where we were searching for lymph nodes in areas within $20 \mathrm{~mm}$ around the airways and the aortic arch, we here replace these search areas by our atlas. Optionally, the atlas can be virtually extended using the information of the Euclidean distance and Voronoi maps.

All parameters needed for lymph node detection were empirically determined in our previous work [16] on the basis of five contrast enhanced CT images.

Aortic Arch and Pulmonary Artery Segmentation. In detail, we start our detection method by segmenting the aortic arch and the pulmonary artery in a new input CT image to exclude these major vessels from our search area. This is necessary because large shape variations of them cannot and should not be fully recovered by our registration method to maintain a certain degree of regularity. To perform the segmentation task, our group recently developed methods applicable to both contrast enhanced and non-contrast CT $[55,56]$. These methods first apply a series of Hough transforms to outline a few approximate points of the centerlines of the aortic arch and pulmonary artery. Then these points are connected by non-uniform rational B-splines and the splines are precisely aligned to the real centerlines by means of a centerline likelihood image generated from a Euclidean distance transform over a thresholded gradient image, where high gradients represent vessel walls and low gradients the lumen. Finally spheres centered along the centerlines are drawn to fill the lumen.

However, we are also considering to extend our current lymph node atlas to incorporate information on vasculature, so we could utilize it during aortic arch and pulmonary artery segmentation, which will be part of our future work.

Hessian Analysis. Now we resample and transform the atlas into input CT image coordinates to define our search region for lymph nodes. Within the minimum bounding box of this search region along the image axes, we search for blob-like structures, as most lymph nodes are of approximate ellipsoidal shape. This can be achieved by standard techniques based on Hessian eigenvalue analysis. We hence determine the Hessian matrix

$$
H(f)_{\sigma}=\left[\begin{array}{ccc}
\frac{\partial^{2} f}{\partial x^{2}} & \frac{\partial^{2} f}{\partial x \partial y} & \frac{\partial^{2} f}{\partial x \partial z} \\
\frac{\partial^{2} f}{\partial y \partial x} & \frac{\partial^{2} f}{\partial y^{2}} & \frac{\partial^{2} f}{\partial y \partial z} \\
\frac{\partial^{2} f}{\partial z \partial x} & \frac{\partial^{2} f}{\partial z \partial y} & \frac{\partial^{2} f}{\partial z^{2}}
\end{array}\right]
$$

for each voxel $\boldsymbol{x}=(x, y, z)^{T}$ of intensity $I(x, y, z)$ by convolution with the second and cross derivatives of a Gaussian [57] of scale $\sigma$. Using a $\mathrm{QL}$ decomposition, we receive its corresponding eigenvalues $\lambda_{1}, \lambda_{2}, \lambda_{3}\left(\left|\lambda_{1}\right| \leq\left|\lambda_{2}\right| \leq\left|\lambda_{3}\right|\right)$. For each voxel, we compute a "blobness" response measure $[58,59,60] B(\lambda)_{\sigma}$ :

$$
B(\lambda)_{\sigma}= \begin{cases}255 \cdot\left(1-e^{-\frac{R_{A}^{2}}{2 \beta^{2}}}\right) \cdot\left(1-e^{-\frac{S^{2}}{2 \gamma^{2}}}\right) & \text { if } \lambda_{j}<0, j=1 \ldots 3 \\ 0 & \text { otherwise }\end{cases}
$$

with

$$
R_{A}=\frac{\left|\lambda_{2}\right|}{\left|\lambda_{3}\right|} \quad \text { and } \quad S=\sqrt{\lambda_{1}^{2}+\lambda_{2}^{2}+\lambda_{3}^{2}}
$$




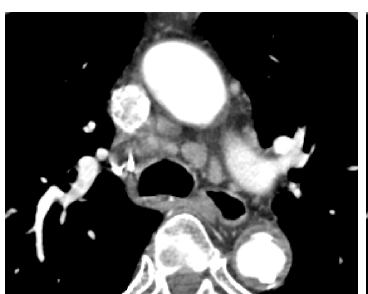

(a) Original image

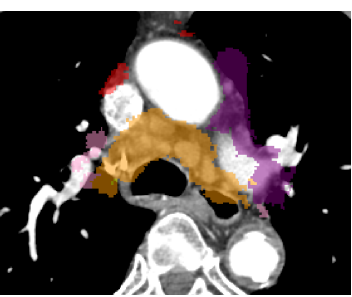

(b) Overlaid atlas

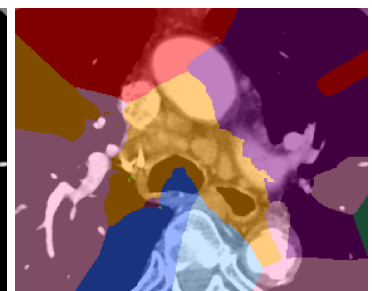

(c) Voronoi map

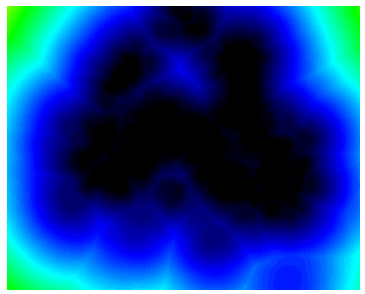

(d) Euclidean distance map

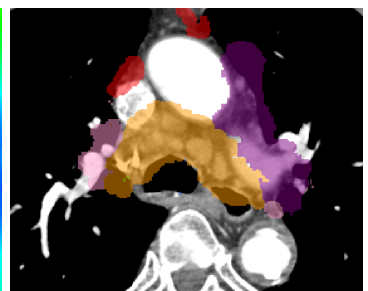

(e) Atlas extended by $2 \mathrm{~mm}$

Figure 5: Atlas extension techniques used for lymph node detection and labeling. If an image (a) is selected from our database and the mediastinal average image and its corresponding atlas (b) are registered to it, the lymph node stations may not be completely covered. However, after creating a Voronoi map (c) of the atlas, every voxel of an image can be labeled, which is important for lymph node station mapping (cf. Sec. 2.3.2). If the Voronoi map is combined with a Euclidean distance map (d) of the atlas, the atlas can also be arbitrarily extended, as shown in (e) for $2 \mathrm{~mm}$ (cf. Sec. 2.3, Atlas Extension). This extension is useful during lymph node detection (cf. Sec. 2.3.1), as the original atlas may not contain all individual lymph nodes of a patient, but is already very close to them.

$B(\lambda)_{\sigma}$ is defined between 0 and 255 and gives high responses for $\lambda_{1} \approx \lambda_{2} \approx \lambda_{3}$. The smaller the eigenvalues, the higher is $B(\lambda)_{\sigma}$. Equally weighting $\beta=\gamma=1$ in Eq. 3 gives a good empirically determined compromise between a low number of true positives and a high number of false positives. $B(\lambda)_{\sigma}$ is computed for multiple scales to obtain blobs of various sizes. We selected these scales as $\sigma=1.5,2.5$, and $3.5(\mathrm{~mm})$, because the minimum long axis of our lymph nodes is about $3 \mathrm{~mm}$ (cf. Sec.3) and our experiments showed that lower scales resulted in many additional small blobs (not being lymph nodes), while adding higher scales recovered no further blobs, but had a much longer runtime. Finally, the maximum response $B(\lambda)$ is selected for each voxel:

$$
B(\lambda)=\max _{\sigma \in\{1.5,2.5,3.5\}} B(\lambda)_{\sigma}
$$

Each lymph node candidate is represented by a blob consisting of 6-connected voxels featuring a blobness measure of at least 1 .

False Positive Reduction. As the Hessian eigenvalue analysis returns many false positive lymph node candidates, we reduce this high number of false positives in the following five steps:

Search Space Restriction Since one important feature of lymph nodes is their typical brightness range and we work on CT images with standard HU, we first threshold the input CT image to only contain voxels inside the intensity interval $[-15,170]$ HU. Furthermore, only voxels inside our atlas based search region and outside all aortic arch and pulmonary artery voxels [16] are considered in the following to reduce the runtime of our algorithm.

Morphological Opening Lymph nodes often touch or infiltrate nearby vessels and soft tissue, which can alter their shape. In order to remove such adjacent small vessels and eliminate other non-spherical structures, we perform morphological opening using a sphere of $1.5 \mathrm{~mm}$ radius (the minimum size of the long axis of lymph nodes; cf. Sec.3) on the thresholded image.
Minimum Volume Thresholding As the minimum volume of lymph nodes is at least about $5.3 \mathrm{~mm}^{3}$ (cf. Sec.3), we remove blobs, which are smaller than $(1.5 \mathrm{~mm})^{3}$.

Directional Difference Filtering Since the extended threedimensional minimum directional difference (3-D MinDD) filter [14] can additionally enhance blobs while suppressing structures with straight boundaries, we apply it to all voxels of candidate blobs (after median filtering the input CT image) to search for blobs of roundish shape:

$$
\begin{aligned}
h(x, y, z)_{r}=\min _{\theta_{1}, \theta_{2}, \phi_{1}, \phi_{2}} 2 I(x, y, z)- \\
\left\{I \left(x+r \cos \left(\theta_{1}\right) \cos \left(\theta_{2}\right),\right.\right. \\
y+r \sin \left(\theta_{1}\right), \\
\left.z+r \cos \left(\theta_{1}\right) \sin \left(\theta_{2}\right)\right)+ \\
I\left(x-r \cos \left(\theta_{1}+\phi_{1}\right) \cos \left(\theta_{2}+\phi_{2}\right),\right. \\
y-r \sin \left(\theta_{1}+\phi_{1}\right), \\
\left.\left.z-r \cos \left(\theta_{1}+\phi_{1}\right) \sin \left(\theta_{2}+\phi_{2}\right)\right)\right\}
\end{aligned}
$$

with $0 \leq \theta_{1} \leq 2 \pi, 0 \leq \theta_{2} \leq 2 \pi,-\frac{\pi}{2} \leq \phi_{1} \leq \frac{\pi}{2}$, and $-\frac{\pi}{2} \leq \phi_{2} \leq \frac{\pi}{2}$ (setting a step size of $\frac{\pi}{8}$ ). For each voxel, the extended 3-D Min-DD filter generates evenly distributed sample points on a sphere centered at the voxel ${ }^{2}$ and for each sample another set of points laying on a hemisphere opposite the sample. Then it compares the intensities of the voxel with the sample points and their corresponding points on the hemispheres. If the intensity of the voxel is higher than the intensities of all sample points, the filter will give a positive response.

We apply the filter to multiple scales using radii $r$ of $1.5,2.0,2.5, \ldots, 8.0 \mathrm{~mm}$, and determine the maximum response:

$$
h(x, y, z)=\max _{r \in\{1.5,2.0,2.5, \ldots, 8.0\}} h(x, y, z)_{r} .
$$

\footnotetext{
${ }^{2}$ The sample points on the sphere are defined by $\theta_{1}$ and $\theta_{2}$ and distributed by geodesic subdivision. For our step size of $\frac{\pi}{8}$ we get 66 sample points.
} 
Blobs not containing any filtered voxels greater than 5 are removed.

Minimum Blobness Thresholding Finally, we remove blobs not containing any voxels with blobness measure greater than an empirically determined threshold. A response measure of 10 gave good results in our experiments. ${ }^{3}$

\subsubsection{Lymph Node Station Mapping}

A second promising application of our probabilistic lymph node atlas is the automatic assignment of lymph node stations to detected or segmented nodes. This can support the physician in evaluating treatment outcomes, comparing results from different groups and institutions, designing and analyzing clinical trials, and planning of patient individual therapy [11].

Once the atlas is transformed into a new CT image with detected or segmented nodes and its Voronoi map is created, we simply assign the corresponding voxels in the Voronoi map to all lymph node voxels. For each lymph node, we now count the number of voxels of all labels and finally label the whole node with the lymph node station that the highest number of voxels is assigned to.

\section{Results}

We evaluated our method on 10 contrast enhanced and 30 non-contrast 3-D chest CT images of various hospitals, scanners, and acquisition parameters. The number of slices of the datasets ranges from 99 to 838 , the slice spacing from 0.4 to 1.5 $\mathrm{mm}$. Each axial slice has $512 \times 512$ pixels of size between 0.5 and $0.665 \mathrm{~mm}$. With the help of a radiologist and various examples illustrating lymph nodes and their corresponding stations in CT images [61, 62, 63, 64], we manually segmented and labeled 847 ground-truth lymph nodes (in average 21.2 nodes per case), which took about one to two hours per case. The radiologist double-checked and confirmed all 847 nodes and their stations. For the reasons mentioned in the introduction, we did not distinguish between malignant and benign nodes, did not assess their pathology, and treated non-contrast and contrast enhanced CT equally. Labeling of station 2 to station 14 nodes was performed according to the latest guidelines [11]. As our datasets did not or only partially cover station 1 lymph nodes, we excluded this station from our experiments.

To get an impression of the size of the lymph nodes we used during our evaluation, we also computed the principal axes and principal moments of each manually segmented lymph node and created an ellipsoid of the same size and of the same axis ratio. In contrast to the RECIST 1.0 guidelines [65], where the long axis diameter was still used to assess lymph nodes, currently the most important criterion for lymph node staging is the length of the short axis of a lymph node, as specified by the RECIST 1.1 guidelines [6]. This is because the short axis is more reproducible than the long axis and a better predictor for

\footnotetext{
${ }^{3}$ Please note that in the original work [16] the minimum blobness threshold was wrongly stated as 30 .
}

the response to chemotherapy and the incidence of metastasis [66]. The short axis of our manually segmented lymph nodes described by the ellipsoid was at least $1.1 \mathrm{~mm}$, at most 38.4 $\mathrm{mm}$, and in average $5.0 \pm 3.6 \mathrm{~mm}$. However, as in some studies still the long axis or the volume of lymph nodes is used for assessment, we also computed these values. The long axis has a minimum of $2.9 \mathrm{~mm}$, a maximum of $80.5 \mathrm{~mm}$, and an average of $13.0 \pm 9.3 \mathrm{~mm}$, and the volume a minimum of $5.3 \mathrm{~mm}^{3}$, a maximum of $80724.2 \mathrm{~mm}^{3}$, and an average of $910.8 \pm 4827.0$ $\mathrm{mm}^{3}$.

To assess the performance of our automated detection and labeling method in a fair way, we applied a leave-one-out strategy to all 40 cases, i.e. when creating the probabilistic lymph node atlas, we excluded the manual segmentations and labels of the current case to be evaluated. We registered the average image to all CT images applying the global image alignment process described in Sec. 2.2.1 and the deformable registration approach described in Sec. 2.1 and transformed the corresponding probabilistic lymph node atlases (generated by the leaveone-out strategy) accordingly.

During deformable registration, we use four image and control point resolution levels, starting with $60 \mathrm{~mm}$ spacing which is then refined to 30,15 , and $7.5 \mathrm{~mm}$. The weighting factor for regularization $\alpha$ is set to 0.5 and the label set scaling factor to $2 / 3$. When utilizing mutual information as similarity measure, we use 32 bins for the histograms, equally distributed over the entire intensity range. All other parameters are set to the default values proposed previously [41], i.e. the maximum allowed displacement of each level of the multi-scale approach is bound to the grid resolution, the sampling rate from the zerodisplacement to the maximum displacement is 5, sparse sampling is used, and 5 optimization cycles are performed on each pyramid level.

\subsection{Atlas Creation}

We created an average image once using mutual information and once using normalized cross correlation as similarity measure during registration. The process stopped after nine iterations. We measured its average runtime on a workstation with two 64-bit Quad Core Intel Xeon 5482 processors. As registration of one image pair took 15 min using mutual information and 2 min using normalized cross correlation and 40 registrations are necessary per iteration, we had an approximate runtime of about 12 hours for mutual information and 1.5 hours for normalized cross correlation, if all eight cores of the CPUs were utilized.

The creation of the corresponding probabilistic atlas took only a few minutes. Figure 4 shows the resulting average image and atlas.

\subsection{Lymph Node Detection}

Table 1 shows the detection results averaged over all 40 data sets in terms of sensitivity and positive predictive value, separated into contrast enhanced, non-contrast, and all data sets. We define the sensitivity and positive predictive value (PPV) as

$$
\text { sensitivity }=\frac{\mathrm{TP}}{\mathrm{TP}+\mathrm{FN}}, \quad \mathrm{PPV}=\frac{\mathrm{TP}}{\mathrm{TP}+\mathrm{FP}},
$$




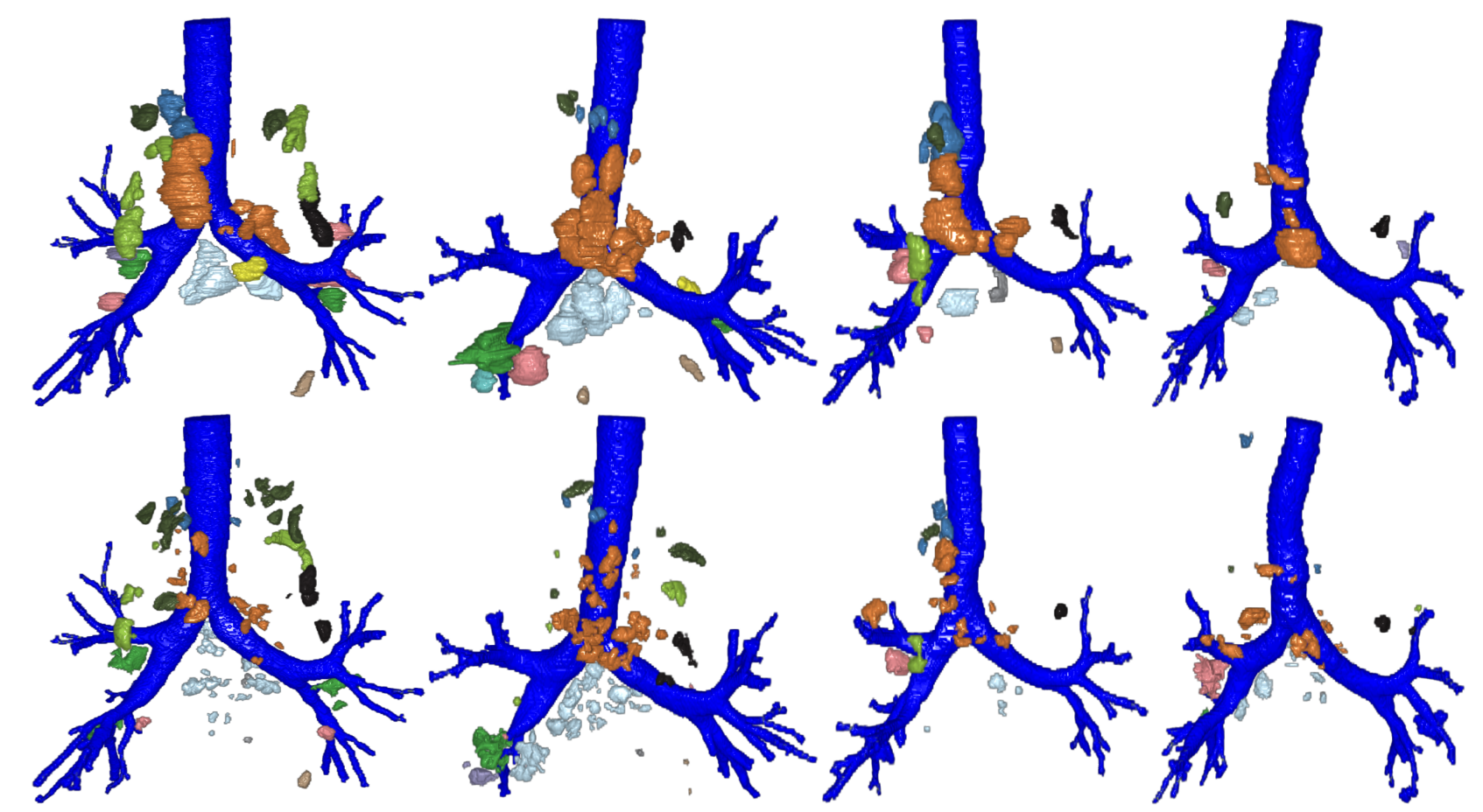

Figure 6: Ground-truth lymph node delineations (top row) compared to automated detection results (bottom row), colored according to Fig. 1. Registration was performed using mutual information, virtually extending the atlas by 2 mm (as highlighted in Tab. 1).

with TP being the number of true positives, FN the number of false negatives, and FP the number of false positives. A groundtruth lymph node is regarded to be detected and thus true positive if at least one of its voxels is covered by one of the extracted blobs. We repeated the experiments for both normalized cross correlation and mutual information as similarity measure and virtually extending the atlas. The extension was only performed for mutual information, as both normalized cross correlation and mutual information gave very similar results.

Table 1: Detection results averaged over all 40 data sets, in terms of sensitivity and positive predictive value (PPV) for contrast enhanced (CE), non-contrast $(\mathrm{N}-\mathrm{C})$, and all data sets, depending on whether normalized cross correlation (NCC) or mutual information (MI) is used as similarity measure and how far the atlas is virtually extended.

\begin{tabular}{l||r|r|r||r|r|r} 
CT Type & CE & N-C & All & CE & N-C & All \\
\hline \hline Method & \multicolumn{2}{|c||}{ Sensitivity [\%] } & \multicolumn{3}{|c|}{ PPV [\%] } \\
\hline \hline NCC, no ext. & 66.5 & 54.2 & 57.0 & 30.4 & 16.3 & 18.7 \\
\hline MI, no ext. & 64.5 & 54.5 & 56.8 & 32.0 & 18.4 & 20.7 \\
\hline MI, 2 mm ext. & 78.2 & 65.5 & 68.5 & 21.1 & 12.7 & 14.2 \\
\hline MI, 4 mm ext. & 81.2 & 69.2 & 72.0 & 16.6 & 10.4 & 11.6 \\
\hline MI, 6 mm ext. & 81.7 & 70.5 & 73.1 & 14.4 & 9.1 & 10.1
\end{tabular}

We also conducted a statistical analysis by a Wilcoxon signed-rank test for the results in Table 1 . Neither the sensitivity nor the PPV between NCC and MI without extension are statistically significant $(p>0.1)$. However, both the sensitivity and the PPV between MI without extension and MI with $2 \mathrm{~mm}$ extension are significant $(p<0.01)$. Those between MI with 2 $\mathrm{mm}$ extension and MI with $4 \mathrm{~mm}$ extension are not $(p>0.05)$.

While Fig. 6 visually compares our automatic detection results to manually segmented lymph nodes, Tab. 2 shows the sensitivity according to the length of the short and long axis of a lymph node. As shown in Tab. 2, our method detects far more bigger than smaller lymph nodes, which is in particular beneficial to detect malignant nodes that are usually enlarged.

Table 2: Number of lymph nodes and sensitivity of lymph node detection according to the axis length of a lymph node. Inwards-pointing brackets indicate inclusion of an endpoint and outwards-pointing brackets its exclusion.

\begin{tabular}{c||r|r|r}
\multicolumn{1}{c||}{ Length [mm] } & Number & Detected & Sensitivity [\%] \\
\hline \hline \multicolumn{4}{c}{ Short Axis } \\
\hline$[0,5[$ & 566 & 318 & 56.2 \\
\hline$[5,10[$ & 235 & 218 & 92.8 \\
\hline$[10,15[$ & 29 & 27 & 93.1 \\
\hline$[15, \infty[$ & 17 & 17 & 100.0 \\
\hline \hline \multicolumn{4}{c}{ Long Axis } \\
\hline$[0,5[$ & 62 & 89 & 14.5 \\
\hline$[5,10[$ & 336 & 186 & 55.4 \\
\hline$[10,15[$ & 225 & 178 & 79.1 \\
\hline$[15,20[$ & 103 & 90 & 87.4 \\
\hline$[20,25[$ & 49 & 46 & 93.9 \\
\hline$[25,30[$ & 27 & 27 & 100.0 \\
\hline$[30, \infty[$ & 45 & 44 & 97.8
\end{tabular}


We also measured the average runtime of our detection method on the same workstation as for average image generation. In detail, airways extraction up to the left and right main bronchi and carina detection takes about $1.5 \mathrm{~min}$, aortic arch extraction $1 \mathrm{~min}$, pulmonary artery segmentation $5 \mathrm{~min}$, global image alignment $15 \mathrm{~s}$, registration using mutual information 15 min and using normalized cross correlation $2 \mathrm{~min}$, respectively, reversing the global image alignment another $15 \mathrm{~s}$, and the actual detection process $2 \mathrm{~min}$. So the overall runtime sums up to about $25 \mathrm{~min}$ for mutual information and $12 \mathrm{~min}$ for normalized cross correlation.

\subsection{Lymph Node Station Mapping}

To evaluate the performance of atlas-based labeling, we removed the lymph node station information from all labels of the manually generated ground-truth data and applied our method to re-label this data set in order to cover all available lymph nodes.

Table 3 shows the sensitivity of the labeling results averaged over the 40 data sets, again separated into contrast enhanced, non-contrast, and all data sets. As for detection, we performed our experiments for both normalized cross correlation and mutual information. As can be seen from the station mapping results, registration using mutual information performs better, in particular for labeling in non-contrast CT.

Table 3: Labeling results averaged over the 40 data sets, in terms of sensitivity for contrast enhanced (CE), non-contrast (N-C), and all data sets.

\begin{tabular}{l||c|c|c} 
CT Type & CE & N-C & All \\
\hline \hline Method & \multicolumn{3}{|c}{ Sensitivity [\%] } \\
\hline \hline NCC & 73.1 & 73.4 & 73.3 \\
\hline MI & 71.6 & 74.9 & 74.1
\end{tabular}

If we consider lymph node labeling as a separate process independently running from detection, we need to perform carina detection $(1.5 \mathrm{~min})$, global image alignment $(15 \mathrm{~s})$, and registration using mutual information (15 min) or normalized cross correlation ( $2 \mathrm{~min}$ ), reverse the global image alignment (15 s), and apply the labeling process, which takes about $1 \mathrm{~min}$. So we have an overall runtime of about $18 \mathrm{~min}$ for mutual information and $5 \mathrm{~min}$ for normalized cross correlation.

\subsection{Dependency of Atlas Generation on Number of Label Im- ages}

In a final evaluation step we also analyzed the dependency of our detection and labeling results on the number of label images used for atlas generation. Evenly distributing labels corresponding to contrast enhanced and non-contrast data, we selected only $5,10,15,20,25,30,35$, and finally 39 label images for atlas generation.

As depicted in Fig. 7 for mutual information and no virtual atlas extension, the sensitivity for both detection and labeling

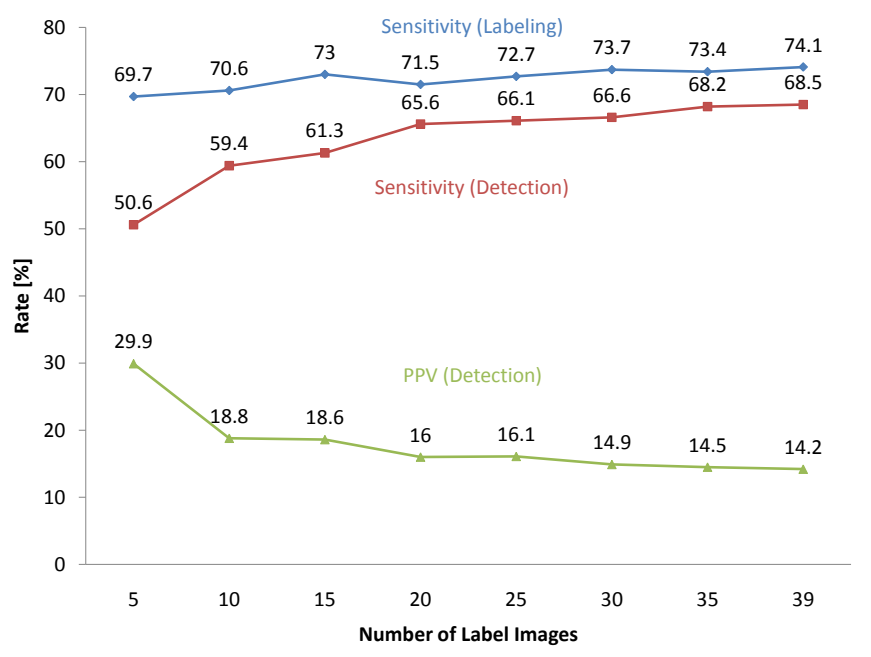

Figure 7: Dependency of detection and labeling results on the number of images used for atlas generation, here shown for mutual information and a virtual atlas extension of $2 \mathrm{~mm}$.

tends to improve with increasing number of label images, so we expect them to further improve if we incorporate more groundtruth segmentations into our atlas. However, for lymph node detection there is always a trade-off between the sensitivity and the positive predictive value in our current method.

\section{Discussion}

In the following we discuss the significance of our results, compare them with other works, and give other relevant considerations.

\subsection{Deformable Registration and Atlas Creation}

As mentioned previously, for deformable registration we use a large number of image and control point resolution levels, iteratively refine our search space, and start with a large spacing between control points. This makes our approach robust against high inter-patient variability. However, there are rare cases where e.g. the aortic arch crosses over the right bronchus and not over the left bronchus. Even though our search space is big and the aortic arch is a very prominent structure in the mediastinum, our registration technique may not successfully address such cases.

Our method can also deal well with contrast intensity differences, as it utilizes illumination-independent similarity measures like mutual information. Since we create an average image of a rather high number of database images, cardiac motion artifacts apparent in single images can also be ruled out sufficiently.

For creation of the average image and registration of all images, we also compared our approach to the one provided by the Image Registration Toolkit (IRTK) ${ }^{4}$ [42], which uses free-form

\footnotetext{
${ }^{4}$ http://www.doc.ic.ac.uk/ dr/software/
} 
deformations, a gradient descent optimizer, and normalized mutual information. For both a paired-T test and a Wilcoxon test, there was no statistical significance $(p>0.1)$ in sensitivity and PPV of lymph node detection between our method and IRTK. However, the runtime of IRTK is significantly longer. It took about three to four hours per registration, compared to at most 15 minutes for our method.

By the nature of our average image creation technique, the average image is blurrier than any of the original database images, as shown in Fig. 8 for two axial slices of the final mediastinal image. This can be seen e.g. at the edges of the aorta and pulmonary arteries. Very small structures such as the pulmonary ligaments cannot be preserved in the average image. Even though they are needed to manually identify pulmonary ligament nodes, surrounding bigger structures such as the pulmonary veins are distinctive and prominent enough and hence sufficient for successful automatic registration and labeling. Since little lymph nodes from other stations are in proximity to the ligament nodes, our approach can still achieve a high labeling sensitivity (also cf. Tab. 5).
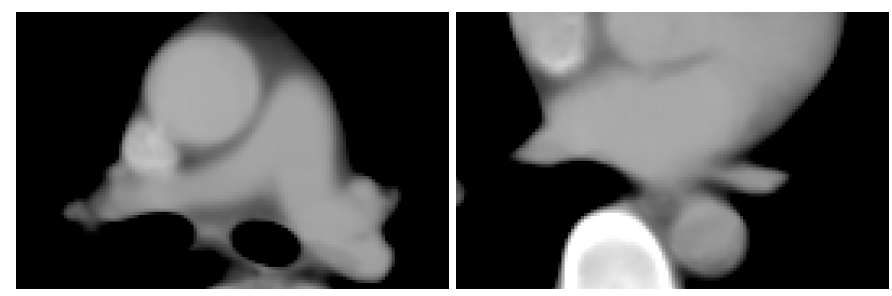

Figure 8: Sample axial slices of the average mediastinal image showing ascending aorta and pulmonary artery (left) and descending aorta and pulmonary veins at the approximate location of the pulmonary ligament (right). The window level is set to $30 \mathrm{HU}$ and the size to $400 \mathrm{HU}$.

It should be noted that for registration we assume the patient to be placed in supine position in the CT scanner, as this is the case for most chest CT acquisitions. However, if the patient was positioned differently, we could identify unique representative anatomic landmarks such as bones [67], estimate the main body axes from these landmarks, and rotate the data accordingly, in addition to the steps done for global image alignment.

\subsection{Lymph Node Detection}

In contrast to $[32,33,34,35,36,37,38,39,40]$, where only lymph node regions are defined or detected, we here present methods for the detection and labeling of single lymph nodes, which can be in particular helpful for planning biopsy paths or assessing disease progression and treatment response.

Our method analyzing the shape of lymph nodes applies five steps to reduce false positives after Hessian analysis. Even though their order is not critical for a successful reduction, it is indeed important to reduce the runtime of our method. For example, directional difference filtering takes a long time and hence should be applied towards the end of our filter pipeline.

We also conducted a statistical analysis by a paired-T test for the long and short axes of manual and automatic segmentations. As expected, both long and short axes of the detected lymph nodes are significantly smaller than those of manually segmented nodes $(p<0.01)$. Table 2 just shows detection sensitivities categorized by the lengths of short and long axes of manually segmented nodes, which were detected by our method. However, we do not think that showing the significance is meaningful, because we do not focus on how accurately lymph nodes are segmented. What we want to show in Tab. 2 is the fact that our method can detect bigger lymph nodes accurately, which are more important in practice than smaller ones.

Our method still misses to detect a certain percentage of lymph nodes. This is mainly due to the detection method itself and not due to registration errors. When looking at the registered data sets a good registration of the mediastinum is clearly visible. Only the hilar, segmental, and subsegmental airways and their patient-specific branching patterns sometimes cannot be registered well, which may lead to a few additional misses.

It can also be seen in Fig. 6 that the lymph node shapes cannot be fully recovered by the detection method. This is because our method assumes lymph nodes to be blob-shaped, even though they sometimes can be of other, more irregular shapes, in particular if they are malignant. Any blobness filter in our pipeline will thus only highlight parts of lymph nodes, which tend to be spherical. This irregularity of the shape of the lymph nodes makes it also difficult to apply other methods to automatic detection such as template matching. One possible approach to tackle this problem may be the integration of a model-based method that seeds a deformable mesh into each highlighted part and adapts this mesh to the real shape of the lymph node, similar to [15].

Furthermore, there is still a number of false positive nodes left. The false positives mainly occur inside the esophagus, vasculature, and musculature. In future work, these areas should be automatically extracted, too, to specify further exclusion areas. In addition, machine learning techniques may be applied to learn intensity and shape feature distributions of lymph nodes and eliminate false positive nodes, as proposed in a recent work [68].

We compared the results to our previous method [16] developed for contrast enhanced CT and having a much larger search space. The results in Tab. 4 clearly show that our atlas can effectively constraint the search space, as the positive predictive value for the same sensitivity doubles. It can also be seen that the aortic arch and pulmonary artery segmentation methods developed for non-contrast CT help to boost the detection rate in comparison to our previous approach solely developed for contrast enhanced CT.

Table 4: Detection results in comparison to our previous method when considering a similar sensitivity for contrast enhanced data, which our previous method was developed for.

\begin{tabular}{l||r|r|r||r|r|r} 
CT Type & CE & N-C & All & CE & N-C & All \\
\hline \hline Method & \multicolumn{2}{|c|}{ Sensitivity [\%] } & \multicolumn{3}{|c|}{ PPV [\%] } \\
\hline \hline No atlas [16] & 77.2 & 57.4 & 62.0 & 13.5 & 5.4 & 6.5 \\
\hline MI, 2 mm ext. & 78.2 & 65.5 & 68.5 & 21.1 & 12.7 & 14.2
\end{tabular}




\subsection{Lymph Node Station Mapping}

We compared the results of our lymph node station mapping approach to a method previously developed by another group [25] for contrast enhanced CT. In [25], machine learning techniques are applied to features collected in segmentations of the airways and the aortic arch such as unit vectors from the lymph nodes to the carina, to bifurcations of the airways, or to the top of the aortic arch, or distances between lymph nodes and the segmented structures. After selection of the most appropriate eight features, station labels can be assigned to manually segmented lymph nodes.

This approach achieves a sensitivity of $76 \%$ on datasets with 8.6 lymph nodes per case in average, while our datasets in average contain 21.2 nodes per case. Our atlas-based labeling method with $73.3 \%$ and $74.1 \%$ for normalized cross correlation and mutual information, respectively, achieves almost the same sensitivity and, contrary to [25], was also evaluated successfully on non-contrast CT.

Please note that in our approach the previously mentioned segmentations of the aortic arch and pulmonary artery are only necessary for the lymph node detection step, but not for labeling. This means that if manual lymph node segmentations are available (as for [25]) our approach will only require a segmentation of the airways for global image alignment (in fact only of the trachea, left and right main bronchus, which all available automatic algorithms achieve in a robust manner), but no aorta segmentation as in [25]. In terms of runtime our approach will take a little longer, as we require an additonal registration step, while [25] only requires an additional segmentation step.

To also get an impression about the strengths and weaknesses of our labeling method, we break down our results according to the lymph node station, as shown in Table 5. It is obvious that in particular stations $8,10,12,13$, and 14 are not labeled well. This is because it is sometimes difficult to distinguish stations 8 and 10 from station 7 , and stations 12,13 , and 14 from station 11 , even by a human observer. Moreover, the further we go down the airways, the more individual they become for each patient, making a good registration difficult or even impossible. Combining our mediastinal atlas with a lung atlas [31] may help here. Another reason may be that we only have very little lymph nodes segmented in these areas. We assume our method to further improve if we incorporate more ground-truth labels.

\section{Conclusion}

In this paper we presented a method to create a mediastinal average image and a novel lymph node atlas containing superior and inferior mediastinal, aortic, and N1 nodes. Combined with a Voronoi map and a Euclidean distance map for arbitrary atlas extension, we successfully applied the atlas to lymph node detection and labeling, resulting in a good sensitivity and positive predictive value compared to previous methods $[16,25]$ at an acceptable runtime.

We would also like to emphasize at this point that our mediastinal lymph node atlas can easily be integrated into other lymph node detection methods suffering from low positive predictive value, making it a valuable tool also for other groups
Table 5: Number of lymph nodes and sensitivity of lymph node labeling according to the lymph node station.

\begin{tabular}{rl||r|r|r}
\multicolumn{1}{l||}{ Station } & Number & Detected & Sen.[\%] \\
\hline \hline 1 & Supraclavicular & \multicolumn{2}{|c}{ insufficient coverage } \\
\hline 2 & Upper Paratracheal & 74 & 56 & 75.7 \\
\hline 3 & Prevasc./Retrotrach. & 111 & 79 & 71.2 \\
\hline 4 & Lower Paratracheal & 261 & 237 & 90.8 \\
\hline 5 & Subaortic & 82 & 78 & 95.1 \\
\hline 6 & Para-aortic & 70 & 41 & 58.6 \\
\hline 7 & Subcarinal & 63 & 61 & 96.8 \\
\hline 8 & Paraesophageal & 21 & 3 & 14.3 \\
\hline 9 & Pulm. ligament & 20 & 18 & 90.0 \\
\hline 10 & Hilar & 33 & 5 & 15.2 \\
\hline 11 & Interlobar & 36 & 35 & 97.2 \\
\hline 12 & Lobar & 51 & 13 & 25.5 \\
\hline 13 & Segmental & 23 & 2 & 8.7 \\
\hline 14 & Subsegmental & 2 & 0 & 0.0
\end{tabular}

working on this topic. Our atlas may also be extended to incorporate ground-truth segmentations of the aortic arch, pulmonary artery, esophagus, and heart to initialize and support segmentation tasks.

Our image database consists of 3-D chest CT scans of Japanese patients, who are in general rather slim and thus have little fatty tissue. In future studies, we need to evaluate, whether the incorporation of CT scans from obese patients decreases the overall registration performance and hence detection and labeling accuracy. However, as seen in the evaluation for average patients, the incorporation of further ground-truth data is expected to further boost the sensitivity of both the detection and labeling process.

\section{Acknowledgments}

This work was supported in part by the JSPS postdoctoral fellowship program for foreign researchers, by a Grant-in-Aid for Science Research funded by MEXT and JSPS, and by a Grantin-Aid for Cancer Research funded by the Ministry of Health, Labour and Welfare, Japan.

\section{References}

[1] World Health Organization, Cancer, Fact sheet $N^{\circ} 297$ (February 2009). URL http: //www. who.int/mediacentre/factsheets/fs297/

[2] A. D. L. Sihoe, A. P. C. Yim, Lung cancer staging, Journal of Surgical Research 117 (2004) 92-106.

[3] I. Sluimer, A. Schilham, M. Prokop, B. van Ginneken, Computer analysis of computed tomography scans of the lung: A survey, IEEE Transactions on Medical Imaging 25 (4) (2006) 385-405.

[4] E. M. Toloza, L. Harpole, F. Detterbeck, D. C. McCrory, Invasive staging of non-small cell lung cancer: A review of the current evidence, Chest 123 (1 suppl) (2003) 157S-166S.

[5] H. I. Pass, Mediastinal staging 2005: Pictures, scopes, and scalpels, Seminars in Oncology 32 (3) (2005) 269-278. doi:10.1053/j.seminoncol.2005.02.014. 
[6] E. Eisenhauer, P. Therasse, J. Bogaerts, L. Schwartz, D. Sargent, R. Ford, J. Dancey, S. Arbuck, S. Gwyther, M. Mooney, L. Rubinstein, L. Shankar, L. Dodd, R. Kaplan, D. Lacombe, J. Verweij, New response evaluation criteria in solid tumours: Revised RECIST guideline (version 1.1), European Journal of Cancer 45 (2) (2009) 228-247.

[7] T. Arita, T. Matsumoto, T. Kuramitsu, M. Kawamura, N. Matsunaga, K. Sugi, K. Esato, Is it possible to differentiate malignant mediastinal nodes from benign nodes by size?, Chest 110 (4) (1996) 1004-1008.

[8] K. L. Prenzel, S. P. Mönig, J. M. Sinning, S. E. Baldus, H.-G. Brochhagen, P. M. Schneider, A. H. Hölscher, Lymph node size and metastatic infiltration in non-small cell lung cancer, Chest 123 (2) (2003) 463-467.

[9] E. F. Patz, Jr, J. J. Erasmus, H. P. McAdams, J. E. Connolly, E. M. Marom, P. C. Goodman, R. A. Leder, M. T. Keogan, J. E. Herndon, Lung cancer staging and management: Comparison of contrast-enhanced and nonenhanced helical CT of the thorax, Radiology 212 (1) (1999) 56-60.

[10] M. Takahashi, N. Nitta, R. Takazakura, Y. Nagatani, N. Ushio, K. Murata, Detection of mediastinal and hilar lymph nodes by 16-row MDCT: Is contrast material needed?, European Journal of Radiology 66 (2008) 287291.

[11] V. W. Rusch, H. Asamura, H. Watanabe, D. J. Giroux, R. Rami-Porta, P. Goldstraw, the Members of the IASLC Staging Committee, The IASLC lung cancer staging project: A proposal for a new international lymph node map in the forthcoming seventh edition of the TNM classification for lung cancer, Journal of Thoracic Oncology 4 (5) (2009) 568-577.

[12] M. Tsuzaka, H. Fujita, M. Uno, T. Hara, Development of an automated detection algorithm for mediastinal lymph nodes on chest X-ray CT images, Japanese Journal of Medical Electronics and Biological Engineering 36 (4) (1998) 343-350.

[13] D. Eicke, Detektion von Lymphknoten in Hals CT-Datensätzen, Master's thesis, Otto-von-Guericke-Universität Magdeburg (2005).

[14] T. Kitasaka, Y. Tsujimura, Y. Nakamura, K. Mori, Y. Suenaga, M. Ito, S. Nawano, Automated extraction of lymph nodes from 3-D abdominal CT images using 3-D minimum directional difference filter, in: N. Ayache, S. Ourselin, A. Maeder (Eds.), MICCAI, no. Part II in LNCS 4792, Springer-Verlag, 2007, pp. 336-343.

[15] L. Dornheim, J. Dornheim, Automatische Detektion von Lymphknoten in CT-Datensätzen des Halses, in: Workshop - Bildverarbeitung für die Medizin (BVM), Berlin, 2008, pp. 308-312.

[16] M. Feuerstein, D. Deguchi, T. Kitasaka, S. Iwano, K. Imaizumi, Y. Hasegawa, Y. Suenaga, K. Mori, Automatic mediastinal lymph node detection in chest CT, in: SPIE Medical Imaging, Vol. 7260, Orlando, Florida, USA, 2009, pp. 72600V-1-72600V-11.

[17] J. Rogowska, K. Batchelder, G. Gazelle, E. Halpern, W. Connor, G. Wolf, Evaluation of selected two-dimensional segmentation techniques for computed tomography quantitation of lymph nodes, Investigative Radiology 31 (3) (1996) 138-145.

[18] D. M. Honea, W. E. Snyder, Three-dimensional active surface approach to lymph node segmentation, in: SPIE Medical Imaging, Vol. 3661, 1999, pp. 1003-1011.

[19] J. Yan, T.-g. Zhuang, B. Zhao, L. H. Schwartz, Lymph node segmentation from CT images using fast marching method, Computerized Medical Imaging and Graphics 28 (1-2) (2004) 33-38. doi:10.1016/j.compmedimag.2003.09.003.

[20] J. Yan, B. Zhao, L. Wang, A. Zelenetz, L. H. Schwartz, Marker-controlled watershed for lymphoma segmentation in sequential CT images, Medical Physics 33 (7) (2006) 2452-2460. doi:10.1118/1.2207133. URL http: //link. aip.org/link/?MPH/33/2452/1

[21] K. Lu, W. E. Higgins, Interactive segmentation based on the live wire for 3D CT chest image analysis, International Journal of Computer Assisted Radiology and Surgery 2 (3-4) (2007) 151-167. doi:10.1007/s11548007-0129-x.

[22] J. Dornheim, H. Seim, B. Preim, I. Hertel, G. Strauss, Segmentation of neck lymph nodes in CT datasets with stable 3D mass-spring models: Segmentation of neck lymph nodes, Academic Radiology 14 (2007) 1389-1399.

[23] D. Maleike, M. Fabel, R. Tetzlaff, H. von Tengg-Kobligk, T. Heimann, H.P. Meinzer, I. Wolf, Lymph node segmentation on CT images by a shape model guided deformable surface method, in: SPIE Medical Imaging, Vol. 6914, 2008, pp. 69141S.1-69141S.8.

[24] M.-P. Jolly, L. Grady, 3D general lesion segmentation in CT, in: ISBI, IEEE, 2008, pp. 796-799.
[25] A. P. Kiraly, D. P. Naidich, L. Guendel, L. Zhang, C. L. Novak, Novel method and applications for labeling and identifying lymph nodes, in: A. Manduca, X. P. Hu (Eds.), Medical Imaging 2007: Physiology, Function, and Structure from Medical Images, Vol. 6511 of Proc. of SPIE, 2007, pp. 651111-1-651111-9. doi:10.1117/12.709413.

[26] K. Lu, S. A. Merritt, W. E. Higgins, Extraction and visualization of the central chest lymph-node stations, in: M. L. Giger, N. Karssemeijer (Eds.), Medical Imaging 2008: Computer-Aided Diagnosis, Vol. 6915 of Proc. of SPIE, 2008, pp. 69151B-1-69151B-15. doi:10.1117/12.768691.

[27] P. Taeprasartsit, W. E. Higgins, System for definition of the central-chest vasculature, in: SPIE Medical Imaging, Vol. 7259, 2009, pp. 725918-1$725918-15$.

[28] Innovative technology for automated lymph node analysis: Definiens will introduce a new image analysis application at RSNA 2008, European Hospital 17 (5) (2008) 26

[29] T. Rohlfing, R. Brandt, R. Menzel, D. B. Russakoff, C. R. Maurer, Jr., Quo Vadis, Atlas-Based Segmentation?, 1st Edition, Vol. 3 of Handbook of Biomedical Image Analysis, Kluwer Academic/Plenum Publishers, 2005, Ch. 11, pp. 435-486.

[30] A. Gholipour, N. Kehtarnavaz, R. Briggs, M. Devous, K. Gopinath, Brain functional localization: A survey of image registration techniques, IEEE Transactions on Medical Imaging 26 (4) (2007) 427-451.

[31] B. Li, G. E. Christensen, E. A. Hoffman, G. McLennan, J. M. Reinhardt, Establishing a normative atlas of the human lung: Intersubject warping and registration of volumetric CT images, Academic Radiology 10 (3) (2003) 255-265.

[32] R. Martinez-Monge, P. S. Fernandes, N. Gupta, R. Gahbauer, Crosssectional nodal atlas: A tool for the definition of clinical target volumes in three-dimensional radiation therapy planning, Radiology 211 (3) (1999) $815-828$.

[33] I. Poon, N. Fischbein, N. Lee, P. Akazawa, P. Xia, J. Quivey, T. Phillips, A population-based atlas and clinical target volume for the head-and-neck lymph nodes, International Journal of Radiation Oncology*Biology*Physics 59 (5) (2004) 1301-1311.

[34] C.-C. Teng, L. Shapiro, I. Kalet, Head and neck lymph node region delineation using a hybrid image registration method, in: 3rd IEEE International Symposium on Biomedical Imaging: Nano to Macro, 2006, pp. 462-465.

[35] S. M. Qatarneh, I.-C. Kiricuta, A. Brahme, U. Tiede, B. K. Lind, Threedimensional atlas of lymph node topography based on the visible human data set, The Anatomical Record (Part B: The New Anatomist) 289B (3) (2006) 98-111.

[36] S. Qatarneh, I. Kiricuta, A. Brahme, M. Noz, B. Ferreira, W. Kim, B. Lind, Lymphatic atlas-based target volume definition for intensitymodulated radiation therapy planning, Nuclear Instruments and Methods in Physics Research A 580 (2007) 1134-1139.

[37] A. Taylor, A. G. Rockall, M. E. B. Powell, An atlas of the pelvic lymph node regions to aid radiotherapy target volume definition, Clinical Oncology 19 (7) (2007) 542-550.

[38] O. Commowick, V. Grégoire, G. Malandain, Atlas-based delineation of lymph node levels in head and neck computed tomography images, Radiotherapy \& Oncology 87 (2) (2008) 281-289.

[39] X. Han, M. S. Hoogeman, P. C. Levendag, L. S. Hibbard, D. N. Teguh, P. Voet, A. C. Cowen, T. K. Wolf, Atlas-based auto-segmentation of head and neck CT images, in: Medical Image Computing and ComputerAssisted Intervention, Vol. 5242 of Lecture Notes in Computer Science, Springer, 2008, pp. 434-441.

[40] S. Gorthi, V. Duay, N. Houhou, M. Bach Cuadra, U. Schick, M. Becker, A. Allal, J.-P. Thiran, Segmentation of head and neck lymph node regions for radiotherapy planning using active contour-based atlas registration, IEEE Journal of Selected Topics in Signal Processing 3 (1) (2009) 135147.

[41] B. Glocker, N. Komodakis, G. Tziritas, N. Navab, N. Paragios, Dense image registration through MRFs and efficient linear programming, Medical Image Analysis 12 (6) (2008) 731-741.

[42] D. Rueckert, L. Sonoda, C. Hayes, D. Hill, M. Leach, D. Hawkes, Nonrigid registration using free-form deformations: application to breast $\mathrm{mr}$ images, IEEE Transactions on Medical Imaging 18 (8) (1999) 712-721.

[43] P. Viola, W. M. Wells, Alignment by maximization of mutual information, International Journal of Computer Vision 24 (2) (1997) 137-154.

[44] F. Maes, A. Collignon, D. Vandermeulen, G. Marchal, P. Suetens, 
Multimodality image registration by maximization of mutual information, IEEE Transactions on Medical Imaging 16 (2) (1997) 187-198. doi: $10.1109 / 42.563664$

[45] J. Pluim, J. Maintz, M. Viergever, Mutual-information-based registration of medical images: a survey, IEEE Transactions on Medical Imaging 22 (8) (2003) 986-1004.

[46] L. Junck, J. G. Moen, G. D. Hutchins, M. B. Brown, D. E. Kuhl, Correlation methods for the centering, rotation, and alignment of functional brain images, The Journal of Nuclear Medicine 31 (7) (1990) 1220-1226.

[47] P. Lo, B. van Ginneken, J. M. Reinhardt, M. de Bruijne, Extraction of airways from CT (EXACT'09), in: Second International Workshop on Pulmonary Image Analysis, London, UK, 2009, pp. 175-189.

[48] T. Kitasaka, K. Mori, J. Hasegawa, J. Toriwaki, A method for extraction of bronchus regions from $3 \mathrm{D}$ chest X-ray CT images by analyzing structural features of the bronchus, FORMA 17 (4) (2002) 321-338.

[49] M. Feuerstein, T. Kitasaka, K. Mori, Automated anatomical likelihood driven extraction and branching detection of aortic arch in 3-D chest CT, in: Second International Workshop on Pulmonary Image Analysis, London, UK, 2009, pp. 49-60.

[50] A. Guimond, J. Meunier, J.-P. Thirion, Average brain models: A convergence study, Computer Vision and Image Understanding 77 (2000) 192-210. doi:10.1006/cviu.1999.0815.

[51] D. Rueckert, A. F. Frangi, J. A. Schnabel, Automatic construction of 3D statistical deformation models of the brain using nonrigid registration, IEEE Transactions on Medical Imaging 22 (8) (2003) 1014-1025.

[52] S. Joshi, B. Davis, M. Jomier, G. Gerig, Unbiased diffeomorphic atlas construction for computational anatomy, NeuroImage 23 (2004) S151S160.

[53] A. Guimond, J. Meunier, J.-P. Thirion, Average brain models: A convergence study, Tech. Rep. 3731, Institut National de Recherche en Informatique et en Automatique, Sophia-Antipolis, France (July 1999). URL http://www.inria.fr/rrrt/rr-3731.html

[54] B. King, R. Döker, S. Meier, H. Shin, M. Galanski, A generalized squared Euclidean distance transform with Voronoi maps, The Insight Journal. URL http://hdl . handle.net/1926/196

[55] M. Feuerstein, T. Kitasaka, K. Mori, Adaptive branch tracing and image sharpening for airway tree extraction in 3-D chest CT, in: Second International Workshop on Pulmonary Image Analysis, London, UK, 2009, pp. 273-284.

[56] M. Feuerstein, T. Kitasaka, K. Mori, Adaptive model based pulmonary artery segmentation in $3 \mathrm{D}$ chest CT, in: SPIE Medical Imaging, San Diego, California, USA, 2010.

[57] G. Farnebäck, C.-F. Westin, Improving deriche-style recursive gaussian filters, Journal of Mathematical Imaging and Vision 26 (3) (2006) 293 299. doi:10.1007/s10851-006-8464-z.

[58] A. F. Frangi, W. J. Niessen, K. L. Vincken, M. A. Viergever, Multiscale vessel enhancement filtering, in: MICCAI, Vol. 1496 of LNCS, 1998, pp. 130-137.

[59] Y. Sato, C.-F. Westin, A. Bhalerao, S. Nakajima, N. Shiraga, S. Tamura, R. Kikinis, Tissue classification based on $3 \mathrm{~d}$ local intensity structures for volume rendering, IEEE Transactions on Visualization and Computer Graphics 6 (2) (2000) 160-180.

[60] L. Antiga, Generalizing vesselness with respect to dimensionality and shape, The Insight Journal. URL http: //hdl . handle.net/1926/576

[61] M. Cymbalista, A. Waysberg, C. Zacharias, Y. Ajavon, M. Riquet, G. Rebibo, P. Grenier, CT demonstration of the 1996 AJCC-UICC regional lymph node classification for lung cancer staging, RadioGraphics 19 (4) (1999) 899

[62] J. P. Ko, E. A. Drucker, J.-A. O. Shepard, C. F. Mountain, C. Dresler, B. Sabloff, T. C. McLoud, CT depiction of regional nodal stations for lung cancer staging, American Journal of Roentgenology 174 (3) (2000) $775-782$.

[63] J.-H. Lee, K. S. Lee, T. S. Kim, C. A. Yi, J. M. Cho, M. H. Lee, Mediastinal and hilar lymphadenopathy: Cross-referenced anatomy on axial and coronal images displayed by using multi-detector row CT, J Korean Radiol Soc. 49 (4) (2003) 285-293.

[64] O. Chapet, F.-M. Kong, L. E. Quint, A. C. Chang, R. K. T. Haken, A. Eisbruch, J. A. Hayman, CT-based definition of thoracic lymph node stations: An atlas from the University of Michigan, International Journal of Radiation Oncology*Biology*Physics 2005 (1) (63) 170-178.
[65] P. Therasse, S. G. Arbuck, E. A. Eisenhauer, J. Wanders, R. S. Kaplan, L. Rubinstein, J. Verweij, M. V. Glabbeke, A. T. van Oosterom, M. C. Christian, S. G. Gwyther, New guidelines to evaluate the response to treatment in solid tumors, JNCI Journal of the National Cancer Institute 92 (3) (2000) 205-216. doi:10.1093/jnci/92.3.205.

[66] E. L. van Persijn van Meerten, H. Gelderblom, J. L. Bloem, RECIST revised: implications for the radiologist. a review article on the modified RECIST guideline, European Radiology 20 (6) (2010) 1456-1467. doi:10.1007/s00330-009-1685-y.

[67] P. Kunz, M. Scholz, B. Haas, T. Coradi, Anatomic orientation in medical images (2008). URL http://www . wipo.int/pctdb/en/wo.jsp?WO=2008014082

[68] K. Mori, Y. Nakamura, T. Kitasaka, M. Ito, S. Nawano, K. Misawa, M. Fujiwara, Automated detection and display of abdominal lymph nodes from CT volumes based on local intensity structure analysis, International Journal of Computer Assisted Radiology and Surgery 5 (Suppl. 1) (2010) S125-S126. 